Meta

Journal des traducteurs

Translators' Journal

\title{
L'hétérolinguisme ou penser autrement la traduction
}

\section{Chiara Denti}

Volume 62, numéro 3, décembre 2017

La traduction littéraire comme création

URI : https://id.erudit.org/iderudit/1043946ar

DOI : https://doi.org/10.7202/1043946ar

Aller au sommaire du numéro

Éditeur(s)

Les Presses de l’Université de Montréal

ISSN

0026-0452 (imprimé)

1492-1421 (numérique)

Découvrir la revue

Citer cet article

Denti, C. (2017). L'hétérolinguisme ou penser autrement la traduction. Meta, 62(3), 521-537. https://doi.org/10.7202/1043946ar
Résumé de l'article

L'hétérolinguisme, en dépit d'une présence quasi constante dans l'histoire de la littérature, n'a été que rarement pris en compte par la traductologie. Si les chercheurs l'envisagent, c'est presque toujours pour en avouer

l'intraduisibilité. Il se présente généralement comme une voie sans issue où la traduction ne peut que se fourvoyer. Mais que se passe-t-il si l'on cesse de le considérer uniquement en tant que problème ? Cet article tente de démontrer en quoi les textes hétérolingues, tout en remettant en cause le présupposé monolingue sur lequel se fonde la conception littéraire traditionnelle, ouvrent à une pensée et à une pratique alternatives de la traduction. Cette étude retrace tout d'abord l'histoire de l'hétérolinguisme littéraire, examine ensuite la relation entre écriture hétérolingue et traduction, et se penche enfin sur le devenir de l'hétérolinguisme en traduction. Partant des romans Temps de chien (2001/2003) de Patrice Nganang et Verre cassé (2005) d'Alain Mabanckou et de leurs versions anglaise, espagnole et italienne, ce dernier temps de l'étude propose une analyse des stratégies de traduction de l'hétérolinguisme. Loin de s'avérer un défi impossible, la traduction peut faire résonner la trame des langues du texte de départ, mais à condition de quitter son paradigme monolingue.
Ce document est protégé par la loi sur le droit d'auteur. L’utilisation des services d’Érudit (y compris la reproduction) est assujettie à sa politique d'utilisation que vous pouvez consulter en ligne.

https://apropos.erudit.org/fr/usagers/politique-dutilisation/ 


\title{
L'hétérolinguisme ou penser autrement la traduction
}

\author{
CHIARA DENTI \\ Università di Bologna, Bologna, Italia-Université Paris Nanterre, Paris, France \\ chiara.denti2@unibo.it
}

\section{RÉSUMÉ}

L'hétérolinguisme, en dépit d'une présence quasi constante dans l'histoire de la littérature, n'a été que rarement pris en compte par la traductologie. Si les chercheurs l'envisagent, c'est presque toujours pour en avouer l'intraduisibilité. Il se présente généralement comme une voie sans issue où la traduction ne peut que se fourvoyer. Mais que se passe-t-il si l'on cesse de le considérer uniquement en tant que problème? Cet article tente de démontrer en quoi les textes hétérolingues, tout en remettant en cause le présupposé monolingue sur lequel se fonde la conception littéraire traditionnelle, ouvrent à une pensée et à une pratique alternatives de la traduction. Cette étude retrace tout d'abord l'histoire de l'hétérolinguisme littéraire, examine ensuite la relation entre écriture hétérolingue et traduction, et se penche enfin sur le devenir de l'hétérolinguisme en traduction. Partant des romans Temps de chien (2001/2003) de Patrice Nganang et Verre cassé (2005) d'Alain Mabanckou et de leurs versions anglaise, espagnole et italienne, ce dernier temps de l'étude propose une analyse des stratégies de traduction de l'hétérolinguisme. Loin de s'avérer un défi impossible, la traduction peut faire résonner la trame des langues du texte de départ, mais à condition de quitter son paradigme monolingue.

\section{ABSTRACT}

Heterolingualism, although a constant element in literary history, has seldom been taken into account in translation studies. Whenever translation scholars have considered it, it has almost always been for positing its untranslatability. Heterolingualism is generally seen as a dead end to which translation must surrender. However, why should it be seen as a problem? This article aims to demonstrate how heterolingual texts, while questioning the monolingual assumption underlying the traditional conception of translation, can open the way to an alternative idea and practice of translation. It first provides a historical overview of literary heterolingualism, then focuses on the relationship between heterolingual writing and translation before looking into the prospects of heterolingualism in translation. The last part of the article investigates strategies in the translation of heterolingual texts by analyzing excerpts from two novels, Temps de chien (2001/2003) by Patrice Nganang and Verre cassé (2005) by Alain Mabanckou, and their English, Spanish and Italian versions. Far from facing an impossible challenge, translation can succeed in representing the interweaving of languages of the source text, provided that it draws away from the monolingual paradigm.

\section{RESUMEN}

El heterolingüismo tiene una presencia casi constante en la historia de la literatura mas la traductología le ha dedicado muy pocos estudios. Cuando los investigadores se interesan en él, casi siempre es para reconocer su intraducibilidad. Generalmente se lo presenta como un callejón sin salida en el que la traducción solo puede ser errónea. Sin embargo, ¿qué ocurre si se deja de considerar que es solo un problema? Este artículo pretende demostrar en qué medida los textos heterolingües abren el camino hacia una reflexión y una práctica alternativas de la traducción, cuestionando al mismo tiempo el supuesto monolingüe en el que se basa la concepción literaria tradicional. En primer 
término, el estudio revisa la historia del heterolingüismo literario. Seguidamente, aborda la relación entre escritura heterolingüe y traducción, antes de esbozar el futuro del heterolingüismo en la traducción. Partiendo de las novelas Temps de chien (2001/2003) de Patrice Nganang y Verre cassé (2005) de Alain Mabanckou, así como de sus versiones en inglés, español e italiano, la última parte del estudio analiza las distintas estrategias de traducción del heterolingüismo. Esta no representa en absoluto un desafío imposible: en la traducción puede resonar la trama de lenguas del texto original, siempre y cuando se deje de lado su paradigma monolingüe.

\section{MOTS CLÉS/KEYWORDS/PALABRAS CLAVE}

hétérolinguisme, littérature, postcolonial, hybridité, (in)traduisibilité heterolingualism, literature, postcolonial, hybridity, (un)translatability heterolingüismo, literatura, poscolonial, hibridez, (in)traducibilidad

\section{L'hétérolinguisme au fil des siècles}

Que l'hétérolinguisme soit bien souvent associé, sinon exclusivement du moins généralement, aux écritures postcoloniales et à celles de la diaspora, ainsi que placé volontiers sous le signe des déplacements, des traversées et de la mobilité du monde contemporain, risque de faire oublier que c'est d'une histoire bien plus longue dont il s'agit ici. Les œuvres littéraires écrites à la croisée des langues ne datent pas d'aujourd'hui, tout comme l'hybridité et le plurilinguisme littéraires ne constituent aucunement des phénomènes nouveaux. Les textes plurilingues ont toujours existé (Forster 1968; Stratford 2008), presque en tout temps et un peu partout, et bien avant l'ère qualifiée de postcoloniale; de même il n'y a pas de genre qui, à un moment ou à un autre de l'histoire littéraire, n'ait été affecté par la rencontre de langues différentes. Aussi les écrivains postcoloniaux ne sont-ils ni les seuls ni les premiers à avoir recours au mélange des langues (Buzelin 2006: 92).

S'il est vrai que le multilinguisme est constamment présent au sein de la littérature, il faut toutefois préciser que son statut n'a pas toujours été le même au long de son parcours séculaire: il est tour à tour un élément répondant aux règles d'un genre spécifique, le résultat d'un choix personnel de l'auteur ou encore un signe d'appartenance à un courant littéraire. De surcroît, selon les périodes historiques, la pratique plurilingue représente tantôt la norme, tantôt l'exception; elle est tantôt appréciée, tantôt méprisée (Elwert 1960: 416; Stratford 2008: 459). Il suffit de porter un regard sur son trajet au fil de l'histoire littéraire pour en prendre conscience: alors que l'usage plurilingue est fort répandu et grandement prisé au Moyen-Âge et jusqu'à la fin du XVII ${ }^{e}$ siècle, c'est vers le XIX ${ }^{\mathrm{e}}$ siècle qu'il se heurte à des résistances (Mackey 1993: 43). C'est à cause de l'idéologie romantique reposant sur la correspondance idéale entre une langue, une littérature, une nation que le plurilinguisme entre dans l'ère du soupçon. Le lien établi entre une nation et une seule et même langue - censée exprimer le mieux le caractère de la nation - rend l'hétérogénéité linguistique coupable d'opérer une trahison envers la littérature nationale et la nation (Mackey 1993: 44). Si écrire dans la langue nationale revient à contribuer à la construction de la nation, prendre le parti inverse, à savoir changer de langue pour faire place à la pluralité langagière, équivaut à rompre la loyauté envers la nation (Grutman 2000). Par la suite, après cette défense de l'unilinguisme, il faut attendre l'époque postcoloniale pour que les cohabitations et les côtoiements linguistiques soient à nouveau valorisés (Meylaerts 2013: 538). 
Quoique la pratique plurilingue ne représente rien d'inédit ni d'exceptionnel comme en témoignent les innombrables exemples multilingues qui émaillent l'histoire littéraire (de La Divine Comédie de Dante au Finnegans Wake de Joyce, ou encore au plus récent Nom de la rose de Eco; sans oublier Pantagruel, de Rabelais au $\mathrm{XVI}^{\mathrm{e}}$ siècle et Guerre et paix de Tolstoï, au XIX ${ }^{\mathrm{e}}$ siècle, pour ne limiter le tour d'horizon qu'aux cas les plus célèbres), l'attention portée par le discours critique à ce phénomène est cependant relativement récente. Les observations de Grutman sont à cet égard fort révélatrices: Grutman note que, dans les années 1980, s'intéresser aux textes littéraires plurilingues était un choix inhabituel; l'intérêt du chercheur pour ce domaine d'étude, perçu comme non conventionnel, avait toujours besoin de justification (Delabastita et Grutman 2005: 11). Cette méfiance à l'égard de l'œuvre plurilingue procède d'une conception strictement monolingue de la littérature. Puisque de tels textes transgressent la loi non écrite du monolinguisme de l'œuvre, ils demeurent longtemps cantonnés aux marges (Grutman 2000: 138) : indignes d'attention de la part du critique, ces textes sont réduits à des anomalies aux allures inquiétantes. Voici ce qu'en dit Elwert dans un article paru en 1960: «si le phénomène du mélange linguistique en littérature a été relativement peu étudié [...] c’est aussi peutêtre parce qu'on l'a considéré comme une anomalie, simple curiosité déconcertante, indigne de l'appréciation esthétique» (Elwert 1960: 410). De pareilles affirmations ne doivent pas étonner. D’ailleurs, en ouverture de son article, Elwert précise que: « dans toute production littéraire, il est naturel que l'œuvre soit écrite en une seule langue, d'un bout à l'autre» (Elwert 1960: 409). Il n'est alors guère surprenant que l'œuvre plurilingue soit volontiers apparentée à un phénomène étrange, et jugée comme étant dépourvue de toute valeur esthétique du fait qu'elle dépasse le cadre supposé naturel.

Aujourd'hui, ces prises de position seraient certainement intenables en raison du changement de regard porté sur les écritures plurilingues. C’est en effet au cours des dernières décennies que l'on a assisté à ce qu'on pourrait qualifier de tournant multilingue, s'accompagnant d'un intérêt grandissant pour la pluralité linguistique (Meylaerts 2006: 2). Afin de prendre la mesure du virage accompli, il suffit de comparer la constatation d'Elwert à celle de Grutman, énoncée des années plus tard. Dans son livre consacré au roman québécois du XIX ${ }^{\mathrm{e}}$ siècle, Grutman déclare dès l'incipit qu'« un texte littéraire est rarement uniforme au point de vue de la langue. Plus souvent qu'on ne le croirait, il est entrelardé d'éléments hétérogènes» (Grutman 1997: 11). Il dresse alors un tableau sensiblement différent; en effet, si l'on s'en tient à ses affirmations, la présence de plusieurs langues dans un seul texte n'implique aucun type d'anomalie. Bien au contraire, Grutman nous invite à considérer l'hétérogénéité linguistique d'un texte littéraire comme normale. Aussi, à la suite de ce renversement de perspective, ces textes marqués par la pluralité de langues deviennent des objets d'étude tout à fait légitimes et, à notre époque, les chercheurs, critiques et éditeurs prêtent une attention particulière au plurilinguisme «au point d'y voir la principale force expressive d'un texte ou d'un courant littéraire» (Buzelin 2006: 92). On est bien loin de la réduction du plurilinguisme à un simple accident. À présent, on reconnaît à ces pratiques d'écriture une force créatrice et innovante. Sans doute ce changement de regard fait-il suite à l'essor aussi bien des théories bakhtinienne et postcoloniale, au sein desquelles la valorisation de l'hybridité linguistique devient une préoccupation majeure, que des réflexions élaborées par Deleuze et Guattari sur les littératures qu'ils ont appelées «mineures». D’après les deux philosophes, la littérature mineure, 
qui n'est pas celle d'une langue mineure mais « celle qu'une minorité fait d'une langue majeure» (Deleuze et Guattari 1975: 29), est la seule à être vraiment «grande» et révolutionnaire en vertu de sa condition de déterritorialisation linguistique. La littérature mineure est celle qui «met en variation» la langue majeure, qui la subvertit et la rend étrangère à elle-même. Cette mise en valeur des processus de déterritorialisation conduit alors à accorder une attention particulière au plurilinguisme. Orientations théoriques auxquelles il faut ajouter, bien évidemment, l'émergence du corpus littéraire postcolonial. Au cours de cette période, la littérature s'enrichit de la production d'écrivains provenant de pays anciennement colonisés qui, dans leurs écrits babéliques, font résonner l'imaginaire de langues différentes.

C'est justement au sein du courant postcolonial qu'une large place est accordée au plurilinguisme: envisagé comme un moyen de détourner et de s'approprier les langues impériales imposées, le phénomène plurilingue devient un objet d'étude privilégié dès la publication du travail fondateur de cette perspective critique. C'est, en effet, à l'intérieur du volume collectif The Empire Writes Back, paru en 1989, qu'un chapitre tout entier est précisément consacré aux stratégies d'écriture plurilingues. Quelques années plus tard, ces mêmes techniques sont placées au centre de la réflexion de Zabus qui, dans son livre The African Palimpsest, s'interroge sur les divers procédés employés par les écrivains pour «indigéner» la langue européenne. Mais c'est aussi en dehors de cette mouvance que le plurilinguisme fait l'objet d'enquêtes et de travaux. Nous évoquerons notamment le travail dirigé par Gauvin qui, comme en témoigne son titre, Les Langues du roman. Du plurilinguisme comme stratégie textuelle, est consacré à dévoiler les stratégies textuelles mises en œuvre dans l'espace romanesque pour rendre compte du plurilinguisme (Gauvin 1999). C'est également le cas des études de Simon concernant ceux qu'elle appelle les «trafiqueurs» de langues, dont l'écriture est fortement marquée par les «effets de traduction", les interférences et l'alternance des langues (Simon 1994). Mais surtout, il faut mentionner les recherches développées par Grutman, qui l'ont amené à forger le néologisme «hétérolinguisme» pour désigner «la présence dans un texte d'idiomes étrangers, sous quelque forme que ce soit, aussi bien que de variétés (sociales, régionales, chronologiques) de la langue principale» (Grutman 1997: 37). Le choix du préfixe hétéro-, en lieu et place des propositions existantes qui signalent la présence de plusieurs langues (pluri-, multi-), indique précisément que c'est la différence des langues en présence et non leur nombre qui est important. Entre ces deux préfixes (pluri- et multi-), presque synonymes, il n'existe qu'une différence de degré. En effet, à la différence de multi- qui évoque la cohabitation de nombreuses langues, pluri- se réfère à la présence de plus de deux langues (ou parfois de plus d'une langue). Les deux préfixes sont liés en ce qu'ils mettent l'accent sur la quantité.

L'invention grutmanienne a l'ambition théorique de proposer une approche nouvelle: d'abord elle veut souligner le caractère strictement textuel du phénomène, à la différence de notions telles que bilinguisme ou diglossie associées respectivement à l'individu et à la société; ensuite elle revendique la nécessité de dépasser les cadres d'analyse conventionnels, qui expliquent les faits de langue représentés dans les œuvres littéraires exclusivement en termes de reproduction mimétique de la réalité externe. Il s'agit, en somme, d'aller au-delà du cadre de l'esthétique de la représentation. Enfin et surtout, ce néologisme a le mérite de proposer une conception véritablement alternative de la langue car la différence dont il est question se rencontre non 
seulement entre les langues mais aussi au sein d'une seule et même langue (Suchet 2014). C’est précisément à partir de cette image de langue comme étant constitutivement hétérogène que l'on peut envisager autrement la traduction. En d'autres termes, c'est parce qu'elle occasionne une faille dans la prétendue homogénéité des langues qu'elle interroge la conception traditionnelle de la traduction. Mais si celle-ci est troublée par les textes hétérolingues, c'est également parce qu'ils font ressortir la dimension créatrice de la traduction. Puisque l'hétérolinguisme active un processus créatif, ces textes invalident l'argument selon lequel la traduction n'est qu'une pâle copie de l'original. La traduction n'est plus une démarche incompatible avec la créativité: bien au contraire, elle apparaît comme un travail de création, et non une simple opération de reproduction d'un texte antérieur.

\section{De l'écriture hétérolingue à la traduction, et à rebours}

Les textes qui mettent en jeu plusieurs langues sont étroitement liés à la traduction. Cette mise en relation peut sembler contradictoire - étant donné que le texte hétérolingue est précisément un texte qui refuse et nie la traduction - mais elle se révèle toutefois fort pertinente. Depuis la célèbre définition donnée par Rushdie des écrivains postcoloniaux comme étant des «hommes traduits» (Rushdie 1992/1993: 28), l'écriture postcoloniale a souvent été rapprochée de la traduction.

S'appuyant sur l'étymologie latine du terme traduire (porter de l'autre côté, transporter), la critique emploie la traduction comme métaphore de l'écriture postcoloniale $\mathrm{du}$ fait que celle-ci opère un transfert à travers des frontières à la fois linguistiques et culturelles. Tymoczko, quant à elle, écarte le sens étymologique du terme (évoqué par Rushdie) et pose une analogie entre la pratique des auteurs postcoloniaux et la traduction littéraire interlinguistique: d'après elle, la démarche de l'écrivain postcolonial s'apparente à la tâche du traducteur. Néanmoins, Tymoczko ne manque pas de mettre au jour les différences qui les séparent. Tout d'abord, l'écrivain postcolonial - à la différence du traducteur - se heurte à la transposition non pas d'un texte mais d'un substrat culturel; ensuite, alors que l'écrivain a la possibilité de choisir les éléments à transposer, le traducteur se trouve soumis à de plus nombreuses contraintes à cause de la fidélité imposée par l'original. La troisième et dernière différence tient au fait que le traducteur dispose d'un espace paratextuel beaucoup plus vaste où insérer ses commentaires et ses explications que celui généralement accordé à l'écrivain (Tymoczko 1999: 19-21). En dépit de ces divergences, il est sans doute vrai que les deux pratiques d'écriture se recoupent, dans la mesure où l'écrivain postcolonial, tout comme le traducteur, met en œuvre une transposition d'éléments à la fois linguistiques et culturels. Puisque l'écrivain postcolonial traduit et $s e$ traduit, rien ne semble distinguer ces écritures auctoriales de traductions d'une œuvre unilingue dont elles affichent les marques formelles et les procédés. Comme l'énonce clairement Tymoczko: «both involve similar representations and transposition of language and culture, both types of writing show similar formal patterns " (Tymoczko 2000: 148).

Le même parallèle découle des recherches de Bandia: à ses yeux aussi, l'écriture postcoloniale est le résultat d'une opération de «traduction-écriture» (Bandia 2001: 123). Cette pratique traduisante s'utilise à partir d'un texte source à la fois réel et imaginaire. Cet original est réel en ce que l'écrivain transpose en langue européenne une tradition orale bien vivante, mais il est aussi imaginaire puisque cette source 
originale ne correspond concrètement à aucun texte: il se trouve plutôt fragmenté en plusieurs versions sans qu'aucune ne revendique le statut d'original, qui supplanterait ainsi les autres. Le processus de traduction se réaliserait à deux niveaux de transfert: interlinguistique, étant donné qu'il y a un passage d'une langue (africaine) à une autre (européenne), aussi bien qu'intermédial du fait qu'il y a une transposition de l'oral à l'écrit. Le texte issu de cette pratique traduisante n'en sort pas indemne. Comme l'a relevé Bandia, nombreux sont les procédés qui témoignent de cette double opération de traduction: calques, glissements sémantiques et collocationnels, innovations lexicales, insertion des langues vernaculaires et bien d'autres dispositifs laissent deviner le travail de traduction sous-jacent (Bandia 1993).

Même si l'on s'éloigne du cadre critique pour s'intéresser à la position des écrivains, on achoppe sur la même image traductive de l'écriture postcoloniale. La mise en parallèle avec la traduction est tout aussi récurrente sous la plume des écrivains: nombreux sont les auteurs qui rapprochent leur travail d'écriture de la traduction. D’après l'écrivain, penseur et critique marocain Khatibi (1938-2009), le texte maghrébin est un lieu habité par une traduction sans fin qui s'opère entre la «langue maternelle» et la langue étrangère. Cet espace textuel est le règne de la «bi-langue» donnant lieu à un perpétuel mouvement entre les langues au point que l'acte de lecture devient lui-même traduction (Khatibi 1981: 8). Ainsi, dans sa pratique d'écriture, Okara se voit contraint de jouer le rôle du traducteur:

As a writer who believes in the utilization of African ideas, African philosophy and African folklore and imagery to the fullest extent possible, I am of the opinion that the only way to use them effectively is to translate them almost literally from the African language native to the writer into whatever European language he is using as his medium of expression. (Okara 1963: 15-16)

Il est également indispensable de mentionner la célèbre déclaration de Kourouma qui, à propos de Les Soleils des Indépendances, avoue sans ambages qu'il «a traduit le malinké en français» (Badday 1970: 7).

S’il est vrai que l'écriture hétérolingue gagne à être pensée par analogie avec la traduction, l'inverse n'en est pas moins vrai comme le note Suchet: «il semble donc légitime de penser que si la traduction est une métaphore imparfaite de l'écriture hétérolingue, cette dernière peut en revanche s'avérer fort utile pour repenser les modèles de la traduction» (Suchet 2009: 10). Il suffit de renverser la mise en parallèle et d'envisager alors la traduction à partir de l'écriture hétérolingue, ce qui amène à penser la traduction autrement.

Les théories de la traduction ont négligé pendant longtemps les œuvres hétérolingues. Derrida, dans un article paru en 1985, signalait déjà que «la possibilité pour des langues d'être impliquées à plus de deux dans le texte» était rarement prise en compte par la traductologie (Derrida 1985: 215). Même si ce grand silence théorique est parfois interrompu, l'hétérolinguisme est simplement abordé en tant que problème susceptible de compliquer le processus de traduction au point de le mettre en péril; il suscite un certain pessimisme chez les théoriciens qui proclament souvent l'intraduisibilité de ces œuvres. Même pour Berman - et on ne peut manquer d'en être étonné -, l'hétérogénéité linguistique voue la traduction à l'échec: incapable de relever ce défi, et dès lors de surmonter «l'épreuve de l'étranger", la traduction aboutirait le plus souvent à effacer la superposition de langues (Berman 1985: 79). La défiance bermanienne s'explique par le fait que la définition de la traduction, telle 
qu'elle est couramment acceptée, s'avère incompatible avec toute forme de plurilinguisme. Pour Grutman, si la traduction «involves a substitution of one language for another", tandis que le multilinguisme «evokes the co-presence of two or more languages (in a given society, text or individual)», nous sommes à l'évidence aux prises avec deux démarches antithétiques (Grutman 2004: 157). Il semble que la traduction - du moins d'après cette conception - soit inconciliable avec la pluralité linguistique: puisqu'elle aboutit à remplacer une langue par une autre, cela implique fatalement l'effacement de toute trace plurilingue. Derrida partage la même conviction, voyant dans l'hybridité linguistique un impossible défi pour la traduction. Il parvient à cette conclusion à partir de l'analyse de Finnegans Wake de Joyce et de Pierre Ménard de Borges. S'agissant du roman de Joyce, il se penche sur la phrase And he war et se demande: "dans quelle langue ceci est-il écrit?» Et s'il affirme que la langue anglaise est sans conteste la langue dominante, il précise aussitôt que «le mot allemand (war) pèse sur le mot anglais». Il est impossible, selon lui, de transférer cette coprésence en traduction. Voici ce qu'il en dit: «on ne pourrait pas traduire l'événement qui a consisté à greffer plusieurs langues dans un seul corps». La réécriture borgesienne du Quichotte, quant à elle, fait émerger un questionnement semblable, étant donné qu'il s'agit d'un texte écrit en espagnol mais marqué par le français. Le fait que la traduction française soit vouée à faire disparaître cette superposition de langues pousse Derrida à un aveu d'impossibilité: «la traduction peut tout, sauf marquer [...] cette différence de système de langues inscrite dans une seule langue; à la limite elle peut tout faire passer [...] sauf le fait qu'il y a, dans un système linguistique, peut-être plusieurs langues» (Derrida 1982: 134).

Il est possible de montrer, en revanche, que les textes hétérolingues, loin de représenter un échec pour la traduction, constituent au contraire une chance. Ils offrent l'occasion de formuler des modèles alternatifs dépassant le prétendu monolinguisme ainsi que les couples dichotomiques qui régissent le paradigme traditionnel. Mehrez (1992), l'une des premières à avoir reconnu l'impact de l'écriture hétérolingue sur la pratique et la théorie de la traduction, constate que les textes hétérolingues imposent une révision des notions conventionnelles et du modèle tenu pour acquis en traductologie. D'ailleurs, selon la représentation convenue, la traduction est conçue comme se substituant complètement à un texte écrit dans une seule langue source A et visant un public appartenant à une culture A - le remplaçant par un texte en langue cible $\mathrm{B}$, également unique, destiné à un public $\mathrm{B}$ issu d'une culture B. Un tel modèle, comme Meylaerts l'a mis en évidence, se fonde sur le présupposé fictif selon lequel aussi bien les langues que les textes et les lecteurs sont tous, et toujours, rigidement monolingues, ainsi que sur l'idée d'une parfaite équivalence entre l'original et sa traduction (Meylaerts 2006: 5).

Cette représentation ne résiste pas à l'affrontement avec la littérature hétérolingue, apte à invalider la vision duale de la traduction. À l'épreuve de ces textes, les oppositions du type langue de départ/langue d'arrivée, original/traduction, source/ cible, se trouvent fragilisées. Ces écritures tranchent avec cette représentation puisqu'ils donnent à voir de manière flagrante que la langue spécifiée par la définition convenue n'est ni unique ni homogène: elle est au contraire hybride et déclinée au pluriel. Ce qu'on désigne comme langue A présente les traits d'un amalgame linguistique, fluide et mouvant, qu'il est impossible d'inscrire dans le cadre d'une seule et même langue. De plus, par son hybridité, le texte original ouvre une faille dans ce 
modèle idéalisé: il ne s'agit plus d'un texte monolithique et stable, aisément traduisible dans un autre texte parfaitement équivalent. Il y a plus encore: étant donné que le texte source serait lui-même le produit d'un processus de traduction, c'est le concept même d'original qui s'estompe. Et si l'on est dépourvu d'original, c'est aussi une des notions structurant la traduction - c'est-à-dire la fidélité - qui perd tout son sens. Il faut encore mentionner une dernière subversion produite par les écrits hétérolingues: les lecteurs impliqués par ces écritures sont bien différents des lecteurs rigoureusement monolingues qu'on se figure habituellement. Le pacte de lecture proposé par ces textes demande bien souvent un lectorat hybride, se plaçant dans un entre-deux, voire un entre-plusieurs-langues.

Les théoriciens ont par conséquent cherché à élaborer des propositions nouvelles aptes à corriger le modèle traditionnel battu en brèche par les textes hétérolingues (Suchet 2014). Nous pouvons également citer l'approche formulée par Bandia au cours de sa réflexion sur l'écriture africaine en langues européennes. Ce dernier reprend le concept d'hybridité théorisé par Bhabha, qui lui semble plus juste pour décrire les textes dépourvus d'une identité linguistique stable et, de ce fait, impossibles à cantonner à l'intérieur du binarisme source versus cible, fondé, à l'opposé, sur des entités monolithiques (Bandia 2008: 9). Nous pouvons encore mentionner ce qu'Appiah appelle la «thick translation», stratégie qu'il considère comme indispensable pour traduire la littérature africaine (Appiah 1993). Dans ce cas, les théories occidentales qui envisagent la traduction dans un cadre purement linguistique se révèlent inadéquates car il faut que la traduction prenne en charge les aspects socioculturels, essentiels pour une bonne compréhension. C'est pourquoi la traduction se fait dense dans la mesure où elle s'accompagne d'un important paratexte permettant de placer l'œuvre dans son contexte à la fois linguistique et culturel. Adejunmobi introduit quant à elle le terme de «complex translations» pour décrire les romans de Khatibi et les recueils poétiques du poète malgache Jean-Joseph Rabearivelo (1903-1937), qui viennent perturber la représentation convenue de la traduction (Adejunmobi 1998). Cette notion rend compte d'un original qui n'est ni homogène ni stable, mais au contraire, mouvant et pluriel, puisque, faute d'adopter une seule langue conformément au modèle traditionnel, il s'installe entre les langues et opère un continuel va-et-vient au point d'imposer la traduction comme mode de lecture. La réflexion engagée par Sakai est également exemplaire: en questionnant la dimension homolinguale aussi bien des langues que des communautés linguistiques, le chercheur invite à abandonner un régime (ou mode) d'adresse homolingue et un moyen de communication transparent, pour se tourner vers un régime de traduction hétérolingue. Ce dernier part du principe que la communication peut échouer, que la compréhension n'est pas forcément réciproque, puisque les communautés linguistiques ne sont ni uniformes ni unilingues, et que le moyen linguistique est intrinsèquement hétérogène (Sakai 1997). Voici comment Sakai les définit:

The homolingual address assumes the normalcy of reciprocal and transparent communication in a homogeneous medium so that the idea of translation does not make sense unless a positively heterogeneous medium is involved. In contrast, the heterolingual address does not abide by the normalcy of reciprocal and transparent communication, but instead assumes that every utterance can fail to communicate because heterogeneity is inherent in any medium, linguistic or otherwise. (Sakai 1997: 8) 
Or, non seulement les textes hétérolingues brisent certains présupposés théoriques de la traductologie mais, comme l'a bien montré Buzelin, ils ébranlent aussi quelques idées reçues sur le sujet traduisant et l'opération de traduction (Buzelin 2006). Si l'on part du constat que ces textes demandent un lecteur bilingue ou même plurilingue, l'enjeu est très différent pour le traducteur, auquel la maîtrise de deux langues - celles de départ et d'arrivée - semble ne plus suffire. En raison de leur «bilinguisme radical» (Mehrez 1992: 131), ces textes sont pleinement compréhensibles si l'on fait intervenir une pratique de traduction (dans une des autres langues de l'auteur). Compte tenu du fait que «the language of the Other comes to encode messages which are not readily decoded by the monolingual reader» (Mehrez 1992: 122), cela laisse penser qu'un traducteur monolingue (au sens où il ne possède qu'une seule des langues employées dans le texte) rencontrerait des obstacles tout à fait semblables au cours de son décodage. C'est pour toutes ces raisons que ces textes portent à revoir l'identité du sujet traduisant: ils invitent à laisser la place à un traducteur plurilingue, qui semble le mieux à même de poursuivre cette entreprise traductive. De plus, ces écritures remettent en question la direction couramment acceptée du processus de traduction, à savoir que la traduction fonctionne toujours mieux de la langue étrangère vers la langue mère. Face à la nécessité de restituer les multiples strates exhibées par ces écritures palimpsestes et d'en transmettre les nombreuses allusions culturelles, il semble plus juste d'imaginer un traducteur allant du familier vers l'étranger. Cette exigence de mise en valeur de l'hybridité amène enfin l'écriture hétérolingue à saper un autre fondement de la pratique traductive, c'est-àdire son prétendu caractère individuel. Cela tient au fait que ces textes impliquent une dimension collective du processus de traduction. La collaboration avec des lecteurs provenant du même horizon culturel et linguistique que l'auteur permettrait de saisir ce qui fatalement échappe à un regard uniquement monolingue et monoculturel, dans lequel Mehrez a pressenti, à juste titre, un réel danger (Mehrez 1992).

\section{L'hétérolinguisme en traduction}

Nous nous proposons à présent de nous arrêter sur un tout dernier aspect de la relation entre hétérolinguisme et traduction, à savoir les défis qu'il pose concrètement, un aspect d'autant plus important que la traduction est indissociable de sa pratique. Il faut alors se demander ce qu'il en est du travail linguistique opéré au sein du texte de départ. Quel est le devenir des emprunts, des formes non standards et des formulations qui creusent des écarts dans la langue source?

À cet effet, nous allons examiner quelques extraits du roman Temps de chien de l'écrivain camerounais Patrice Nganang $\left(2001^{1}, 2003^{2}\right)$ et comparer sa version italienne réalisée par Giusy Cutrì ${ }^{3}$ avec la version anglaise d'Amy Baram Reid ${ }^{4}$, ainsi qu'avec la traduction espagnole de Manuel Serrat Crespo ${ }^{5}$. Nous examinerons ensuite le roman Verre cassé du Congolais Alain Mabanckou (20056), et nous mettrons en regard la traduction espagnole effectuée par Mireia Porta i Arnau ${ }^{7}$ avec la traduction anglaise de Helen Stevenson ${ }^{8}$, et les deux traductions italiennes, la première réalisée par Martina Cardelli ${ }^{9}$, la seconde par Daniele Petruccioli ${ }^{10}$.

Ces deux œuvres constituent un lieu d'observation privilégié des stratégies hétérolingues, puisqu'elles déploient une gamme de procédés extraordinairement riche et variée. Ceux-ci sont plus ou moins perceptibles selon les cas: ils sont tantôt éclatants 
et immédiatement visibles (alternance codique, emprunts), tantôt plus discrets et ténus (calques, néologismes de sens). Prenant appui sur les travaux de Suchet, nous observons qu'entre les pôles diamétralement opposés de l'effacement et de la conservation s'ouvrent des choix de traduction intermédiaires, qui interviennent en modifiant la forme visuelle et/ou la lisibilité des occurrences hétérolingues (Suchet: 2014).

C'est ce que montrent les phénomènes d'alternance codique, si nombreux dans Temps de chien, dont on propose ici un exemple suivi de ses traductions:

(1) «Mbe ke di? cria-t-il, mbe ils ont arrêté l'écrivain-a? Sè? Nùm ke? Ntog a ya ? Comment? Vous dites vrai? A tat'te!»

(Nganang 2003: 179; nous soulignons)

(2) «Mbe ke di? cria-t-il, mbe ils ont arrêté l'écrivain-a? Sè? Nùm ke? Ntog a ya? Comment? Vous dites vrai? A tat'te! ${ }^{(73)}{ }^{\prime}$

Note de bas de page:

73. «Quand? Pourquoi? En passant par où? (...) C'est un mensonge!»

(Nganang 2001: 145; nous soulignons)

(a) "Mbe ke di ?" he screamed. "They arrested the writer-a? Sè? When? Why? Which way did they go? How? I don't believe it! A tat'te! It's a lie!"

Glossaire en fin de volume:

Mbe ke di?: What are you saying?

(Nganang 2001/2006: 99, traduit par Reid; nous soulignons)

(b) "Mbe ke di? - gritó -, mbe ellos han detenido al escritor-oh? Se? Num ke? Ntog a ya? ¿Cómo? ¿De verdad? A tat'te!"73

Note de bas de page:

73. “¿Cuándo? ¿Por qué? ¿Pasando por dóndé? (...) ¡Es mentira!”

(Nganang 2001/2010: 147, traduit par Serrat Crespo; nous soulignons)

(c) "Mbe ke di? urlò, mbe loro hanno arrestato lo scrittooore? Sè? Nùm ke? Ntog a ya? Ma come? Dite sul serio? A tat'te!"

(Nganang 2003/2008: 82, traduit par Gutrì; nous soulignons)

Dans ce fragment, où l'on passe du français à une langue vernaculaire (le medùmba), la signification des insertions effectuées dans le texte de départ n'est pas explicitée dans l'édition de poche (exemple [1], 2003), alors qu'une note les explique, au moins en partie, dans la première édition (exemple [2], 2001). Ces notes de bas de page résultaient d'une contrainte éditoriale. Voici comment l'auteur explique leur présence «sous contrainte»:

C'est surtout une question éditoriale [...]. Le Cameroun a 200 langues. Qui a grandi à Yaoundé, a grandi dans un univers où il ne comprendra jamais tout. Il suffit de comprendre la réalité ambiante. Il s'agit d’un processus éditorial qui est surtout français, où on a l'impression que si un mot nous échappe, on est perdu. L'écrivain africain doit encore passer par ce genre de choses. J'espère que le prochain roman ne subira pas cette maltraitance. (Tervonen: 2001)

On note que les insertions subissent d'importantes modifications dans la version anglaise qui se caractérise par une considérable réduction des éléments hétérolingues. Parfois, ils sont complètement effacés lorsque le traducteur opte pour le passage à l'anglais (When? Why? Which way did they go?); parfois, ils sont préservés mais non sans l'accompagnement de leur traduction, soit directement dans le corps du texte 
(la traduction suit immédiatement: it's a lie), soit dans le glossaire en annexe du roman (what are you saying?). Là où le texte de départ - et la traduction espagnole également - refusent de tout éclairer (dans les deux cas la traduction donnée en note est partielle), la version anglaise veut garantir le maximum de compréhension. Cependant, les altérations ne se cantonnent pas à la traduction anglaise: la version espagnole impose, elle aussi, des modifications car, tout comme la traduction anglaise, elle marque les insertions par l'italique. Seule la version italienne les donne sans modification.

De même, lorsqu'on a affaire à l'emprunt, forme hétérolingue la plus fréquemment employée, il peut arriver que l'expression constitue une entrée dans un glossaire et/ou qu'elle soit marquée par la mise en italique, ou encore qu'elle comporte une note. C'est le cas du terme $\underline{k o k \boldsymbol{i}}$ relevé dans Temps de Chien: il désigne une spécialité culinaire et est emprunté à la langue duala. Comme on le voit, dans l'édition de poche l'emprunt reste sans explication, tandis que la première version du roman ainsi que la traduction espagnole en fournissent une définition dans une note. La traduction espagnole le met en italique et la traduction anglaise, non seulement recourt au marquage typographique, mais l'explique aussi dans le glossaire. Ici encore, l'occurrence est reprise sans modification dans la version italienne.

(3) Une fois mon maître demanda à Soumi de me donner une part du délicieux koki.

(Nganang 2003: 32; nous soulignons)

(4) Une fois mon maître demanda à Soumi de me donner une part du délicieux koki. Note de bas de page:

12. Mets aux haricots

(Nganang 2001: 26; nous soulignons)

(a) Una vez mi dueño le pidió a Sumi que me diera parte del delicioso $k o k i^{12}$.

Note de bas de page:

12. Manjar con habichuelas

(Nganang 2001/2010:28, traduit par Serrat Crespo; nous soulignons)

(b) Once my master asked Soumi to give me some of his delicious red and greasy koki.

Glossaire en fin de volume:

Koki: dish made of beans

(Nganang 2001/2006: 17, traduit par Reid; nous soulignons)

(c) Una volta il mio padrone chiese a Soumi di darmi una parte del delizioso koki.

(Nganang 2003/2008: 17, traduit par Cutrì; nous soulignons)

Ces mêmes solutions peuvent en outre figurer là où apparaissent des modifications d'expressions figées, obtenues par l'addition d'un élément qui africanise la locution. On peut citer en exemple la tournure clouer son bec de calao qui a entraîné une large gamme d'attitudes traductives allant de la conservation pure et simple de la traduction espagnole (pico de calao) jusqu'à l'effacement complet de la version anglaise (shut up), en passant par les traductions italiennes: l'une choisit de le conserver tout en ajoutant une explication dans une note et en recourant à l'italique, l'autre opte pour sa substitution (insérant le plus familier tucano).

(5) Je lui ai redit de clouer son bec de calao.

(Mabanckou 2005: 50; nous soulignons) 
(a) I told him again to shut up.

(Mabanckou 2005/2011: 28, traduit par Stevenson; nous soulignons)

(b) Le repetí que cerrara su pico de calao.

(Mabanckou 2005/2007: 39, traduit par Porta i Arnau; nous soulignons)

(c) Gli ho ridetto di chiudere quel becco da calao $^{10}$.

Note de bas de page:

10. Uccello tropicale dal becco lungo e ricurvo [N. d. T]

(Mabanckou 2005/2008: 36, traduit par Cardelli; nous soulignons)

(d) Gli ho detto di chiudere quel becco da tucano.

(Mabanckou 2005/2015: 39, traduit par Petruccioli; nous soulignons)

Au terme de cet aperçu, force est de constater que la conservation suivie de n'importe quelle modification entraîne des effets non négligeables sur le mécanisme hétérolingue. Il est frappant que certaines de ces altérations rompent le pacte de lecture proposé par le texte de départ, car elles font basculer le rapport établi avec le double lectorat impliqué. Cela se produit à l'endroit précis de la confrontation avec l'insertion de gloses explicatives qui se manifestent sous la forme d'un appareil de notes et/ou par le renvoi au glossaire. Ajouter ces explications revient à faciliter la compréhension au lecteur exogène (par rapport à la langue de l'occurrence hétérolingue) qui, en revanche, n'est pas pris en charge par le texte de départ. Puisque les traducteurs présagent qu'il éprouvera des difficultés, ils lui viennent en aide et pallient son dépaysement. Aussi, certains lieux du texte qui se révèlent opaques (au moins aux yeux d'une partie du public francophone) deviennent parfaitement déchiffrables pour le lecteur des versions traduites. Tout se passe ici comme si le lecteur privilégié était le lecteur exogène (européen) et non le lecteur endogène (africain).

Ce bref examen montre que les modifications opérées affectent aussi la représentation des langues en jeu qui, par ces changements, en sortent sensiblement transformées. Cela se produit à la suite de l'ajout du marquage typographique - le recours à l'italique en l'occurrence - qui a pour effet de tenir à distance les occurrences et de les mettre en scène en tant qu'étrangères. Mais c'est sans conteste le fait de les placer dans un glossaire qui souligne leur altérité de manière encore plus manifeste. Il arrive que ce qui se présente comme familier dans le texte de départ (rien ne le distingue de la langue principale) soit traité comme un corps étranger au sein du texte d'arrivée, où il est tantôt soigneusement balisé, tantôt transformé en entrée de glossaire. Si le texte de départ confère aux langues convoquées le même statut, le texte d'arrivée, à l'opposé, crée un écart entre les deux. Ceci n'est pas sans conséquence puisqu'on donne implicitement à entendre que le jugement linguistique du narrateur sur les langues en jeu dans le texte est fort différent: c'est comme si l'une lui appartenait et l'autre pas. Cette démarche rompt avec le texte de départ en ce qu'elle fait fi du rapport de complicité linguistique à l'égard des langues secondes. La disparité de traitement (elles sont mises en italique et donc distanciées) neutralise tout effort pour leur reconnaissance. On est tenté de soupçonner que ces solutions tiennent au fait que la traduction est résolument ancrée dans un imaginaire monolingue: elle tend le plus souvent à supposer tant la langue que le lectorat comme homogènes, uniformes et bien sûr unilingues. Autrement dit, elle adopte volontiers une "adresse homolingue», pour le dire dans les termes de Sakai (2008). Ces postures traductives semblent reposer sur l'idée d'une communauté de réception unique, tout comme sur le postulat 
d'un moyen de communication transparent. Elles sont le signe d'une volonté d'assurer une pleine compréhension, d'éviter au lectorat cible de se heurter à n'importe quel malentendu, effort ou écueil.

Mais pour expliquer de tels choix, il faudrait aussi prendre en compte le lourd conditionnement exercé par l'instance éditoriale. Très souvent, il arrive que les maisons d'édition poussent (ou forcent) les traducteurs vers des options traductives qui vont dans le sens d'une normalisation radicale. C'est le souci d'assurer une lecture facile et agréable - considérée comme un préalable nécessaire à une bonne vente du livre - qui est à l'origine d'attitudes de traduction qui apparaissent tout à fait conformes aux douze «tendances déformantes» âprement dénoncées par Berman (1999: 52-68). C'est en somme, aussi, à la lumière d'un monde éditorial en quête d'une lisibilité et d'une standardisation coûte que coûte (en dépit des caractéristiques affichées par le texte de départ), comme l'a bien montré Venuti, qu'il faut lire ces stratégies de traduction. Un monde éditorial qui s'efforce de laisser «le lecteur le plus tranquille possible» - pour reprendre la célèbre distinction formulée déjà en 1813 par Schleiermacher (1813/1999: 49) -, qui fait tout ce qu'il peut pour que son lectorat se sente à l'aise et qui rend n'importe quel texte accessible et familier, même ceux qui se refusent à l'être. Nous ne pouvons passer sous silence que ces approches traductives risquent d'occulter les dimensions sémantique, politique et esthétique dont sont bien souvent dotés les dispositifs hétérolingues. Elles risquent d'en fausser les visées, qu'il faudrait se garder de toujours vouloir réduire à une simple motivation mimétique car sous la surface de ces techniques d'écriture se cachent bien souvent des enjeux majeurs (identitaires, politiques, culturels, etc.).

La traduction est tout aussi susceptible de réactiver les fonctions à la fois esthétiques et politiques accomplies par l'hétérolinguisme. Mais elle le fait en empruntant une autre voie, à contre-courant de la logique monolingue, puisqu'elle n'obéit plus au diktat de la limpidité à tout prix et défend les zones d'ombre. C'est précisément ce que donne à voir ce passage tiré de la version espagnole de Verre cassé:

(6) Et alors, entre cet arbre et moi s'établissaient de longs causers comme dirait un Nègre à son amiral à qui il apporte de l'eau de café.

(Mabanckou 2005: 192; nous soulignons)

(a) Y entonces entre el árbol y yo se establecían largos charlares, como diría un negro a su almirante al que lleva el agua para café.

(Mabanckou 2005/2007: 131, traduit par Porta i Arnau; nous soulignons)

(b) And the tree and I would have these long conversations, as the negro would say to his admiral when bringing the water for his coffee.

(Mabanckou 2005/2011: 25, traduit par Stevenson; nous soulignons)

(c) Così io e quell'albero facevamo lunghe chiacchierate, come direbbe un negro al suo ammiraglio al quale sta portando un caffè.

(Mabanckou 2005/2008: 127, traduit par Cardelli; nous soulignons)

(d) E allora io e l'albero intavolavamo lunghe conversazioni, come direbbe un negro al suo ammiraglio mentre gli porta l'acqua per il caffè.

(Mabanckou 2005/2015: 141, traduit par Petruccioli; nous soulignons)

Ici, contrairement à ce qui se passe dans les autres traductions, le contrat d'une compréhension intégrale et la règle d'une parfaite transparence sont transgressés: le choix de la forme non standard largos charlares au lieu du familier et attendu largas 
charlas opacifie la lecture exactement comme longs causers dans le texte de départ (où on emploie le verbe causer comme substantif au lieu de l'escompté causerie). Tout en reproduisant tel quel le procédé de transcatégorisation à l'œuvre dans le texte de départ (on observe la même utilisation du verbe - charlar - en fonction de substantif), la traductrice préserve l'éloignement de la norme, qui est entièrement résorbée dans les autres versions. C'est également ce qu'on peut lire derrière la solution adoptée dans la version italienne de Temps de chien pour traduire l'expression tuer les journées, calque des langues véhiculaires. Voici le passage avec ses trois traductions:

(7) Le vendeur de cigarettes avec qui mon maître tuait ses journées.

(Nganang 2001:32; nous soulignons)

(a) The cigarette vendor, with whom my master killed time each day.

(Nganang 2001/2006: 36, traduit par Reid; nous soulignons)

(b) El vendedor de cigarillos con quien mi dueño mataba su tiempo.

(Nganang 2001/2010: 56, traduit par Serrat Crespo; nous soulignons)

(c) Il venditore di sigarette con il quale il mio padrone ammazzava le giornate.

(Nganang 2003/2008: 32, traduit par Cutrì; nous soulignons)

Nous observons que dans les traductions anglaise et espagnole la tournure calquée disparaît pour laisser place aux expressions codifiées (kill time, matar el tiempo). Opter pour l'expression la plus idiomatique est le signe évident de la volonté de normaliser le texte de départ. Alors que ces deux traductions ne veulent pas désorienter leur public, la version italienne échappe à la tendance standardisante: elle opère un calque du calque. Ainsi, le lecteur italophone se heurte comme son homologue francophone à l'effet d'étrangeté suscité par le calque: à la place de l'expression toute faite ammazzare il tempo, son attention est attirée sur la forme déviante ammazzare le giornate. Non seulement ces solutions vont à l'encontre de l'« easy readibility» attaquée par Venuti (1998), mais, comme le veut la version française, elles continuent à dessiner une image de la langue comme étant hétérogène et étrangère à elle-même, et à s'adresser à un lectorat pluriel.

Si l'on jette un regard d'ensemble sur les exemples sélectionnés, on s'aperçoit qu'ils semblent confirmer les réflexions formulées par Venuti en ce qui concerne l'espace éditorial anglo-américain. Ces passages montrent que la tendance normalisante est une constante des traductions anglaises. Ici, les traducteurs ont adopté des stratégies répondant au régime fondé sur la lisibilité et la compréhensibilité, tant dénoncé par Venuti. De plus, les exemples cités illustrent clairement que l'hétérolinguisme se manifeste sous un grand nombre de formes. Alors que certaines manifestations entraînent une légère déstabilisation du système de la langue de départ (notamment l'emprunt et l'alternance codique), car elles se limitent à insérer des segments de longueur plus ou moins importante en langue étrangère, il y en a d'autres qui sont bien plus perturbantes (comme le calque et la transcatégorisation). Cette différence de nature entre les procédés explique - au moins en partie - la diversité des solutions proposées. Notons que si dans les cas de l'emprunt et de l'alternance codique toutes les traductions analysées restituent l'hétérolinguisme (certes pas toujours intégralement), nous ne pouvons en affirmer autant en ce qui concerne les dispositifs du calque et de la transcatégorisation. On ne peut ignorer qu'une seule des quatre traductions que nous avons comparées opte pour la conservation (respective- 
ment la version espagnole pour ce qui se réfère au calque et la version italienne pour ce qui est de la transcatégorisation). Cela tient sans doute au fait que les emprunts et les mélanges de langues se prêtent aisément à l'ajout de l'accompagnement paratextuel, qui est en mesure d'atténuer la charge dérangeante de l'hétérolinguisme. Mais il nous semble aussi que ce décalage a quelque chose à voir avec le divers degré d'hétérolinguisme. À la différence des autres formes, le calque et la transcatégorisation travaillent la langue de départ, modifient les combinaisons convenues et produisent des altérations de la norme. Ainsi, on peut légitimement suggérer l'hypothèse que la plupart des traductions les ont résorbées parce qu'elles sont bien plus déstabilisantes et affichent un plus haut degré d'hétérolinguisme.

Enfin, un dernier aspect - crucial pour la relation de la traduction à la création - mérite qu'on l'examine. Il faut en effet relever que les versions s'opposant à la standardisation mettent en place un processus de création: en recourant à l'invention, elles sont parvenues à préserver l'hétérolinguisme. Dans nos romans, nous retrouvons ce processus de traduction-création à plusieurs occasions. Prenons, à titre d'exemple, deux passages tirés de la traduction italienne de Temps de chien:

(8) Après avoir laissé se perdre le sans-payer au loin [...].

(Nganang 2003: 82; nous soulignons)

(a) Dopo aver lasciato il mezzo a sbafo perdersi lontano [...].

(Nganang 2003/2008: 39, traduit par Cutrì ; nous soulignons)

(9) «Un mange-mille, je vous assure!»

(Nganang 2003: 88; nous soulignons)

(a) "Un mangia-grana, ve lo dico io".

(Nganang 2003/2008: 42, traduit par Cutrì; nous soulignons)

Dans les deux cas, la mise en place de l'invention permet à la traductrice de restituer la richesse de la matière langagière du texte de départ. L'écart du français hexagonal que provoquent les deux néologismes - sans-payer et mange-mille (qui désignent respectivement le fourgon de la police camerounaise et l'agent qui exige la somme de mille francs lors des contrôles des taxis) - est rendu visible par les créations lexicales (mezzo a sbafo et mangia-grana), qui sont aptes à produire le même effet de défamiliarisation du texte source.

\section{Conclusion}

$\mathrm{Au}$ terme de cette enquête nous pouvons nous accorder sans peine sur ce que les traductologues constatent depuis longtemps, à savoir que l'hétérolinguisme complexifie grandement la traduction en ce qu'il élève au carré l'altérité du texte de départ. L'étrangeté de la langue de départ se trouve accrue par la présence d'autres langues qui lui sont étrangères. Les textes hétérolingues possèdent une indéniable dimension problématique, comme le remarque Berman : «la prolifération babélienne des langues pose des questions de traduction spécifiques» (Berman 1999: 52). Cela dit, il faut admettre que l'hétérolinguisme ne peut plus être vu tout simplement comme un problème. Il faut essayer de mieux poser la question et l'appréhender plutôt en tant qu'occasion de renouvellement de la pratique traduisante. Car, non seulement l'hétérolinguisme ouvre la possibilité de penser la traduction en dehors 
du modèle monolingue, mais il invite également à la considérer comme un acte créatif et innovant. Comme nous l'avons vu, il agit comme un moteur de créativité susceptible d'ouvrir les portes à l'imagination du traducteur. La traduction devient un espace de créativité: ce n'est qu'en mettant en œuvre un processus de création linguistique, une traduction-création, que le texte traduit parvient à véhiculer l'univers langagier du texte de départ.

\section{NOTES}

1. Nganang, Patrice (2001): Temps de chien. Paris: Le Serpent à Plumes.

2. Nganang, Patrice (2003): Temps de chien. Paris: Le Serpent à Plumes.

3. Nganang, Patrice (2003/2008): Tempi da cane. (Traduit par Giusy Cutrì) Turin: Tirrenia Stampatori.

4. Nganang, Patrice (2001/2006): Dog Days. (Traduit par Amy Baram Reid) Charlottesville/ Londres: University of Virginia Press.

5. Nganang, Patrice (2001/2010) : Tiempo de perro. (Traduit par Manuel Serrat Crespo) Barcelone: El Aleph Editores.

6. Mabanckou, Alain (2005): Verre cassé. Paris: Seuil.

7. Mabanckou, Alain (2005/2007): Vaso roto. (Traduit par Mireia Porta i Arnau) Barcelone: Alpha Decay.

8. Mabanckou, Alain (2005/2011): Broken glass. (Traduit par Helen Stevenson) Londres: Serpent's Tail.

9. Mabanckou, Alain (2005/2008): Verre cassé. (Traduit par Martina CARdelli) Milan: Morellini.

10. Mabanckou, Alain (2005/2015): Pezzi di vetro. (Traduit par Daniele Petruccioli) Rome: 66thand2nd.

\section{RÉFÉRENCES}

Adejunmobi, Moradewun (1998) : Translation and Postcolonial Identity: African Writing and European Languages. The Translator. 4(2):163-181.

Appiah, Kwame A. (1993): Thick Translation. Callaloo. 16(4):808-819.

Aschсroft, Bill, Griffiths, Gareth et Tiffin, Helen (1989): The Empire Writes Back. Theory and Practice in Post-colonial Literatures. New York: Routledge.

BADDAY, Moncel (1970): Ahmadou Kourouma, écrivain africain. Afrique littéraire et artistique. 10:2-8.

Bandia, Paul (1993): Translation as Culture Transfer: Evidence from African Creative Writing. TTR. 6(2):55-78.

Bandia, Paul (2001): Le concept bermanien de l'«Étranger» dans le prisme de la traduction postcoloniale. TTR. 14(2):123-139.

BANDiA, Paul (2008): Translation as Reparation: Writing and Translation in Postcolonial Africa. Manchester: St. Jerome.

Berman, Antoine (1985): La traduction comme épreuve de l'étranger. Texte: Traduction: Textualité / Text: Translatability. 4:67-81.

Berman, Antoine (1999): La Traduction et la lettre: ou l'Auberge du lointain. Paris: Seuil.

Buzelin, Hélène (2006) : Traduire l'hybridité littéraire: Réflexions à partir du roman de Samuel Selvon: The Lonely Londoners. Target. 18(1):91-119.

Delabastita, Dirk et Grutman, Rainier (2005): Introduction. Fictional representations of multilingualism and translation. In: Dirk Delabastita et Rainier Grutman, dir. Fictionalising Translation and Multilingualism. Linguistica Antverpiensia. 4:11-34.

Deleuze, Gilles et Guattari, Félix (1975): Kafka. Pour une littérature mineure. Paris: Éditions de Minuit.

DeRrIDA, Jacques (1982): L'oreille de l'autre: otobiographies, transferts, traductions. In: Claude LÉvesque et Christie V. Mcdonald, dir. Textes et débats avec Jacques Derrida. Montréal: VLB, 125-212. 
Derrida, Jacques (1985): Des Tours de Babel. In: Joseph F. Graham, dir. Difference in Translation. Ithaca/Londres: Cornell University Press, 209-248.

ELWERT, Wilhelm T. (1960): L'emploi des langues étrangères comme procédé stylistique. Revue de littérature comparée. 34(3):409-437.

Forster, Leonard (1970): The Poet's Tongues: Multilingualism in Literature. Londres/New York/ Sidney: Cambridge University Press.

Gauvin, Lise (1992): L'imaginaire des langues: entretien avec Édouard Glissant. Études françaises. 28(2-3):11-22.

Gauvin, Lise, dir. (1999): Les langues du roman. Du plurilinguisme comme stratégie textuelle. Montréal: Presses de l'Université de Montréal.

Grutman, Rainier (1997): Des langues qui résonnent l'hétérolinguisme au XIXe siècle québécois. Québec: Fides.

Grutman, Rainier (2000) : Écriture bilingue et loyauté linguistique. Francophonie d'Amérique. $10: 137-147$.

Grutman, Rainier (2004): Multilingualism and Translation. In: Mona BAKer, dir. Routledge Encyclopedia of Translation Studies. Londres/New York: Routledge, 157-160.

Khatibi, Abdelkebir (1981) : Lettre-Préface. In: Marc Gontard. La violence du texte. La littérature marocaine de langue française. Paris: L'Harmattan, 7-9.

Mackey, William (1993) : Literary Diglossia: Biculturalism and Cosmopolitanism in Literature. Visible Language. 27(1-2):40-66.

Menrez, Samia (1992): Translation and Postcolonial Experience: the Francophone North African text. In: Lawrence Venuti, dir. Rethinking Translation, Discourse, Subjectivity, Ideology. New York: Routledge, 120-138.

Meylaerts, Reine (2006): Heterolingualism in/and translation: How legitimate are the Other and his/her language? An Introduction. Target. 18(1):1-15.

Meylaerts, Reine (2013): Multilingualism as a challenge for translation studies. In: Carmen Millán et Francesca Bartrina, dir. The Routledge Handbook of Translation Studies. Londres: Routledge, 537-551.

OKara, Gabriel (1963): African Speech... English Words. Transition. 10:15-16.

Rushdie, Salman (1992/1993): Patries Imaginaires. Essais critiques, 1981/1991. (Traduit par Aline Chatelin) Paris: Christian Bourgois.

SAKAI, Naoki (1997): Translation and Subjectivity. Minneapolis: University of Minnesota Press.

SCHLeIERMACher, Friedrich (1813/1999): Ueber die Verschiedenen Methoden des Uebersezens / Des différentes méthodes du traduire. (Traduit par Antoine Berman et Christian Berner) Paris: Seuil.

Simon, Sherry (1994): Le trafic des langues: traduction et culture dans la littérature québécoise. Montréal: Boréal.

Stratford, Madeleine (2008): Au tour de Babel! Les défis multiples du multilinguisme. Meta. 53(3):457-470.

Suchet, Myriam (2014): L'imaginaire hétérolingue. Ce que nous apprennent les textes à la croisée des langues. Paris: Classiques Garnier.

Tervonen, Taina (Dernière mise à jour: $1^{\mathrm{er}}$ avril 2001): L'écrivain à l'école de la rue. Entretien avec Patrice Nganang. Page consultée le 17 août 2016, www.africultures.com/php/?nav=article $\&$ no $=2009$.

Tүмосzко, Maria (1999): Post-colonial writing and literary translation. In: Susan BAssnetT et Harish Trivedi, dir. Postcolonial Translation Theory and Practice. Londres: Routledge, $19-40$.

Тумосzко, Maria (2000): Translations of Themselves: the contours of postcolonial fiction. In: Sherry Simon et Paul St-Pierre, dir. Changing the Terms. Ottawa: Presses de l'Université d'Ottawa, 147-163.

Venuti, Lawrence (1998): The Scandals of Translation: Towards an Ethics of Difference. New York: Routledge.

Zabus, Chantal (1991): The African Palimpsest. Indigenization of Language in the West African Europhone Novel. Amsterdam: Rodopi. 